\title{
More efficient induction of antitumor $T$ cell immunity by exosomes from CD40L gene-modified lung tumor cells
}

\author{
JIAOLI WANG ${ }^{1 *}$, LIMIN WANG $^{1 *}$, ZHENDONG LIN $^{2 *}$, LISHA TAO $^{3}$ and MING CHEN ${ }^{4}$ \\ ${ }^{1}$ Department of Respiratory Medicine, Hangzhou First People's Hospital, Hangzhou, Zhejiang 310006; Departments of \\ ${ }^{2}$ Orthopedics, ${ }^{3}$ Gastroenterology and ${ }^{4}$ Otolaryngology, The Second Affiliated Hospital, \\ Zhejiang University School of Medicine, Hangzhou, Zhejiang 310058, P.R. China
}

Received June 12, 2013; Accepted October 8, 2013

DOI: $10.3892 / \mathrm{mmr} .2013 .1759$

\begin{abstract}
The incidence of lung cancer increases annually. However, the effects of the present methods for the treatment of lung cancer are extremely poor. It has been reported that exosomes from heat-stressed 3LL Lewis lung tumor cells effectively elicit systemic antitumor immunity. CD40 signaling is critical in the activation of dendritic cells (DCs), which are important in the induction of antitumor immunity. In the present study, exosomes from CD40 ligand gene-modified 3LL tumor cells (CD40L-EXO) were identified to be more immunogenic compared with control-EXO and lac Z-EXO. CD40L-EXO induced a more mature phenotype of the DCs and promoted them to secrete high levels of interleukin-12. CD40L-EXO-treated DCs induced a greater proliferation of allogeneic $\mathrm{T}$ cells in the mixed lymphocyte reaction. Moreover, CD40L-EXO induced robust tumor antigen-specific CD4 ${ }^{+}$ $\mathrm{T}$ cell proliferation ex vivo. CD40L-EXO were also extremely effective in the protective and therapeutic antitumor tests in vivo. These results indicate that CD40L-EXO may be used as an efficient vaccine for lung cancer immunotherapy.
\end{abstract}

Correspondence to: Dr Jiaoli Wang, Department of Respiratory Medicine, Hangzhou First People's Hospital, 22 Huansha Road, Hangzhou, Zhejiang 310006, P.R. China

E-mail: wangj1230@sina.com

*Contributed equally

Abbreviations: DCs, dendritic cells; CD40L, CD40 ligand; MLR, mixed lymphocyte reaction; CTLs, cytotoxic T lymphocytes; TEX, exosomes derived from tumor cells; Ad-CD40L, recombinant murine CD40L adenovirus; CD40L-EXO, exosomes from Ad-CD40L-infected 3LL lung cancer cells; Lac Z-EXO, exosomes from Ad-Lac Z-infected 3LL lung cancer cells; Control-EXO, exosomes from uninfected 3LL lung cancer cells; Ad, adenovirus; BMDCs, bone marrow-derived DCs

Key words: exosomes, CD40, dendritic cells, lung cancers

\section{Introduction}

The morbidity and mortality of lung cancer has been rapidly increasing over the past 50 years. The incidence of lung cancer has accounted for $12 \%$ of all new cancer cases, and lung cancers are the second most common malignancies in individuals (1). Surgical intervention is the most effective method to treat lung cancer; however, the recurrence following surgery remains high. Moreover, the effect of traditional radiation and chemical treatment on pulmonary carcinoma is extremely limited. It is therefore necessary to urgently find a novel method to treat lung cancers more efficiently.

Exosomes are lipid bilayer vesicles, $30-90 \mathrm{~nm}$ in diameter, formed within late endocytic compartments and physicochemically distinct from other secreted vesicles (2-4). Exosomes may be released from various living cells, including tumor cells, dendritic cells (DCs), platelets, cytotoxic T lymphocytes (CTLs) and reticulocytes (2,5). Exosomes derived from tumor cells (TEX) contain tumor antigens, and a number of studies have indicated that exosomes may be used as an effective vaccine to initiate antitumor immunity $(6,7)$. However, the immunogenicity of the tumor antigen in TEX is poor and the efficiency of TEX to induce antitumor immunity requires further improvement. One of the most significant characteristics of exosomes is their suitability for artificial modification. It has been reported that TEX from heat-stressed tumor cells or super-antigen-anchored TEX are more immunogenic and may induce more efficient tumor antigen-specific CTL responses $(8,9)$. It may be assumed that TEX is likely to be an attractive vaccine for the prevention of tumors. TEX from heat-stressed 3LL lung tumor cells are effective in the regression of the established lung cancer in mice, which indicates that TEX are also effective in the treatment of lung cancers.

CD40, a member of the tumor necrosis factor receptor superfamily, is expressed on B cells, monocytes and DCs. CD40 is significant in the improvement of immune responses following ligation with its natural ligand, CD40 ligand (CD40)/CD154 (10). DCs are unique professional antigen-presenting cells that prime naïve $\mathrm{T}$ cells. DCs are considered to be the most attractive candidates in the activation of immunity against cancer (11). The activation of CD40 signaling in DCs may promote their differentiation and matu- 
ration, and the secretion of immunostimulatory cytokines, including interleukin (IL)-12, which is significant in the activation of the antitumor $\mathrm{T}$ cell response $(12,13)$. However, CD40L is predominantly expressed on activated $\mathrm{T}$ cells, but not on resting $\mathrm{T}$ cells $(14,15)$, which possibly restricts the activation of DCs. It was hypothesized that TEX from CD40L gene-modified 3LL cells are likely to be more efficient in the activation of DCs and the subsequent antitumor $\mathrm{T}$ cell immunity.

In the present study, the antitumor effect of exosomes derived from CD40L gene-modified 3LL tumor cells was investigated. Recombinant murine CD40L adenovirus (Ad-CD40L) was constructed, and then CD40L gene-modified 3LL tumor cells (CD40L-EXO) from Ad-CD40L-infected 3LL lung cancer were isolated. The CD40L-EXO were demonstrated to be more efficient in the induction of mature DCs and IL-12 secretion than TEX from Ad-Lac Z-infected (Lac Z-EXO) and uninfected 3LL tumor cells (Control-EXO). CD40L-EXO were more efficient in improving the ability of DCs to present antigens than Lac Z-EXO and Control-EXO. Moreover, DCs pulsed with CD40L-EXO significantly increased tumor antigen-specific $\mathrm{CD} 4^{+} \mathrm{T}$ cell proliferation and CTL responses. Notably, CD40L-EXO revealed a more powerful antitumor effect than Lac Z-EXO and Control-EXO in vivo.

\section{Materials and methods}

Reagents. $\mathrm{CD}^{+} \mathrm{T}$ cell isolation kit II was purchased from Milteny Biotec (Bergisch Gladbach, Germany) and the ELISA kit with IL-2, IL-12p70, TNF- $\alpha$ and interferon (INF) $-\gamma$ and phycoerythrin (PE)-conjugated antibodies against CD40 (1c10), MHCII (M5/114.15.2), CD80 (16-10A1) and CD86 (D03.1) was obtained from eBioscience (San Diego, CA, USA). The PE-conjugated antibodies against CD40L (MR1) were obtained from BD Biosciences (San Diego, CA, USA). The antibodies against HSP70 (F-3), CD63 (Y-8), CD9 (KMC8.8) and TSG101 (c-2) were purchased from Santa Cruz Biotechnology, Inc. (Santa Cruz, CA, USA). The lactose dehydrogenase (LDH) assay kit was purchased from Promega Corporation (Wisconsin, CA, USA). The aldehyde/sulfate latex beads and the alamarBlue cell proliferation assay kit were from Invitrogen Life Technologies (Carlsbad, CA, USA).

Cell lines and mice. The 3LL Lewis lung cancer and B16 melanoma cell lines were obtained from the American Type Culture Collection (Manassas, VA, USA). The 3LL and B16 cells were maintained in RPMI-1640 media and Dulbecco's modified Eagle's medium (DMEM; Hyclone, Rockford, IL, USA), respectively, supplemented with $10 \%$ fetal calf serum (FCS; Hyclone). C57BL/6 mice were obtained from Joint Ventures Sipper BK Experiment Animals Co. (Shanghai, China). Female mice, 6-8 weeks old, were housed in a specific pathogen-free facility. The experimental protocols were approved by the Animal Care and Use Committee of the School of Medicine, Zhejiang University (Hangzhou, China).

Construction of recombinant mouse Ad-CD40L. Ad-CD40L was constructed using the AdMax ${ }^{\mathrm{TM}}$ system (Microbix Biosystems, Mississauga, OR, Canada). Briefly, the DNA fragment for murine CD40L was amplified from splenocytes of the C57BL/6 mice by polymerase chain reaction using the following specific primers: Sense: 5'-GCCGAATTCATGATA GAAACATACAGCCAA-3' and antisense: 5'-GACGTCGAC GAGTTTGAGTAAGCCAAAAGA-3', synthesized by Sangong Biotech Shanghai Co. Ltd. (Shanghai, China). The CD40L sequence was then inserted into a pDC315 shuttle vector and co-transfected with pBHGlox $\Delta \mathrm{E} 1,3 \mathrm{Cre}$ Ad backbone plasmid (transfected with pBHGlox $\triangle \mathrm{E} 1$ and backbone plasmid) into HEK293 cells to generate Ad-CD40L. The generated Ad-CD40L was purified and stored at $-80^{\circ} \mathrm{C}$.

Ad infection and exosome purification. For Ad infection, the 3LL cells were infected with Ad-CD40L or Ad-Lac Z for $24 \mathrm{~h}$. The cells were then washed intensively five times and incubated with fresh medium for $48 \mathrm{~h}$. The cell-culture supernatants were centrifuged at a series of speeds $(300 \mathrm{x} \mathrm{g}$ for $10 \mathrm{~min}, 1,200 \mathrm{x} \mathrm{g}$ for $20 \mathrm{~min}$ and $10,000 \mathrm{x} \mathrm{g}$ for $20 \mathrm{~min}$ ) to remove the cell debris, the centrifuged supernatant was aggregated through ultracentrifugation $(100,000 \mathrm{x} \mathrm{g}$ for $1 \mathrm{~h})$ and the pellets were run on a $30(1.13)$ to $45 \%(1.19 \mathrm{~g} / \mathrm{ml})$ discontinuous sucrose density gradient $(100,000 \mathrm{x} \mathrm{g}$ for $2 \mathrm{~h}$ ) to purify the exosomes. The middle layer was collected and subjected to another ultracentrifugation to obtain the purified exosomes.

Electron microscopy. Exosomes were fixed in $4 \%$ paraformaldehyde, loaded onto formvar carbon-coated electron microscopy grids (Phillips Electronic Instruments, Mahwah, NJ, USA), contrasted and embedded in a mixture of uranyl acetate and methylcellulose. Sections were observed with a Philips Tecnai-10 transmission electron microscope operating at $80 \mathrm{kV}$ (Phillips Electronic Instruments).

Western blot analysis. In total, $10 \mu \mathrm{g}$ exosomal proteins were separated by a $10 \%$ SDS-polyacrylamide gel and transferred onto a polyvinylidene difluoride membrane. Following incubation with anti-HSP70 (F-3), CD63 (Y-8), CD9 (KMC8.8) and TSG101 (c-2) antibody and corresponding horseradish peroxidase-coupled secondary antibody sequentially, the specific band on the membrane was visualized with a chemiluminescence kit (ECL detection kit, Amersham Bioscience, Piscataway, NJ, USA).

DC generation and fluorescence-activated cell sorting (FACS) analysis. Bone marrow cells from the C57BL/6 mice were cultured in RPMI-1640 medium containing 10\% FCS, $10 \mathrm{ng} / \mathrm{ml}$ granulocyte macrophage-colony stimulating factor and $1 \mathrm{ng} / \mathrm{ml} \mathrm{IL-4}$ to generate bone marrow-derived DCs (BMDCs). Following culture growth for a period of five days, $1 \times 10^{6} / \mathrm{ml}$ BMDCs were stimulated with 1 or $10 \mu \mathrm{g} / \mathrm{ml}$ CD40L-EXO, LacZ-EXO and Control-EXO or $1 \mu \mathrm{g} / \mathrm{ml} \mathrm{lipo-}$ polysaccharide (LPS) for $48 \mathrm{~h}$. For FACS analysis of the BMDCs, $1 \times 10^{6}$ BMDCs were incubated with the corresponding PE-conjugated antibodies for $20 \mathrm{~min}$.

For the FACS analysis of the exosomes, $20 \mu \mathrm{g}$ exosomes were incubated with $5 \mu \mathrm{l} 4-\mu \mathrm{m}$ diameter aldehyde/sulfate latex beads for $15 \mathrm{~min}$ at room temperature in a $20-\mu \mathrm{l}$ final volume, followed by gentle agitation for $1 \mathrm{~h}$ in $1 \mathrm{ml}$ phosphate-buffered saline (PBS) and centrifugation at 3,000 x $\mathrm{g}$ for $5 \mathrm{~min}$. The 

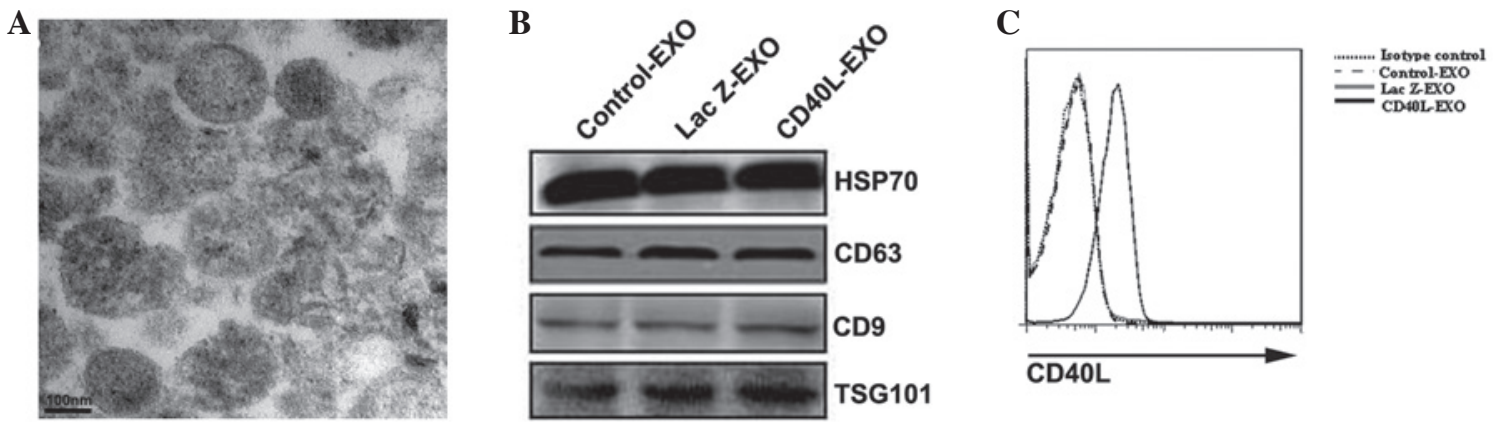

Figure 1. Characterization of CD40L-EXO. Electron microscopy analysis of CD40L-EXO. (A) CD40L-EXO were isolated by differential centrifugation, washed and visualized by electron microscopy. Scale bar, $100 \mathrm{~nm}$. (B) Western blot analysis of characteristic exosome proteins; $10 \mu \mathrm{g}$ exosomal proteins were analyzed by western blot analysis for the presence of several exosome characteristic proteins. (C) Exosomes were absorbed onto latex beads, washed and then stained with anti-CD40L. The CD40L carried on the exosomes was detected by fluorescence-activated cell sorting. Data are representative of three independent experiments. CD40L-EXO, CD40 ligand gene-modified 3LL tumor cells.

pellet was blocked by incubation with $20 \mu \mathrm{FCS}$ for $30 \mathrm{~min}$. Exosome-coated beads were washed three times in PBS and then resuspended in $50 \mu \mathrm{l}$ PBS. The beads were incubated for $1 \mathrm{~h}$ with the corresponding fluorescent antibodies. The cells and beads were analyzed by flow cytometry using a FACS Calibur flow cytometer and CellQuest software (Becton-Dickinson, Mountain View, CA, USA).

Cytokine assay. The levels of IL-12p70 and tumor necrosis factor (TNF)- $\alpha$ from the exosome-stimulated BMDCs and the levels of IFN- $\gamma$ and IL-2 from the splenocytes of the exosome-immunized C57BL/6 tumor mice ex vivo were detected by ELISA kits according to the manufacturer's instructions.

Mixed lymphocyte reaction (MLR). The BMDCs from the C57BL/6 mice were stimulated with $10 \mu \mathrm{g} / \mathrm{ml}$ CD40L-EXO, LacZ-EXO or Control-EXO, or $1 \mu \mathrm{g} / \mathrm{ml}$ LPS for $48 \mathrm{~h}$. The BMDCs were then collected and inactivated by $50 \mu \mathrm{g} / \mathrm{ml}$ mitomycin $\mathrm{C}$ for $30 \mathrm{~min}$ at $37^{\circ} \mathrm{C}$. CD $4^{+} \mathrm{T}$ cells from the splenocytes of the $\mathrm{BALB} / \mathrm{c}$ mice were isolated using a $\mathrm{CD} 4^{+} \mathrm{T}$ cell isolation kit II (Miltenyi Biotec, Surrey, UK). BMDCs and CD4 ${ }^{+} \mathrm{T}$ cells were co-cultured at the indicated ratio for $72 \mathrm{~h}$. AlamarBlue was then added per well for an additional culture for $24 \mathrm{~h}$. The fluorescence intensities were detected to determine the $\mathrm{CD} 4^{+}$ $\mathrm{T}$ cell proliferation.

Antigen presentation assay. The C57BL/6 mice were immunized four times at an interval of seven days by $100 \mu \mathrm{g}$ 3LL cell lysates. At 35 days post-immunization, the $\mathrm{CD}^{+} \mathrm{T}$ cells derived from the splenocytes were isolated and co-cultured with syngeneic BMDCs that had been pre-treated with 1 or $10 \mu \mathrm{g} / \mathrm{ml}$ exosomes for $48 \mathrm{~h}$. Following co-culture for $72 \mathrm{~h}$, the proliferation of the $\mathrm{CD} 4^{+} \mathrm{T}$ cells was also detected by the alamarBlue assay.

Immunization and tumor challenge. The C57BL/6 mice were immunized subcutaneously (s.c.) into the left flank by CD40L-EXO, LacZ-EXO, Control-EXO $(10 \mu \mathrm{g} / 100 \mu \mathrm{l}$ PBS/mouse) or $100 \mu \mathrm{l}$ PBS 13 days before 3LL cell challenge. The immunization was boosted three times on days seven, nine and 11 , respectively. On day 0 , the immunized mice were challenged with $1 \times 10^{6} 3 \mathrm{LL}$ cells s.c. The tumor size was measured and the survival rate of the tumor mice was recorded on the indicated days. To induce IL- 2 and IFN- $\gamma, 5 \times 10^{6} / \mathrm{ml}$ splenocytes from mice seven days after the last immunization were stimulated with inactivated 3LL cells at a ratio of 10:1 for $48 \mathrm{~h}$.

To investigate the therapeutic effect of CD40L-EXO, the 3LL tumor model was established with $1 \times 10^{6} 3 \mathrm{LL}$ cells per mouse. Exosomes were then injected (10 $\mu \mathrm{g} / \mathrm{mouse})$ with on days $+7,+9,+11$ and +13 . The tumor size was measured and the survival rate of the tumor mice was also recorded on the indicated days.

Cytotoxic assay of CTL. For the cytotoxic assay, splenocytes were obtained from the immunized mice. The splenocytes were stimulated with inactivated 3LL cells and with IL-2 $(30 \mathrm{U} / \mathrm{ml})$ for seven days. The 3LL cells were used as the specific target cells, while B16 cells were used as the control target cells and pulsed splenocytes were used as the effector cells. The ratios of effector to target were 12.5:1, 25:1 and 50:1. The cells were co-cultured for $4 \mathrm{~h}$ and then the cytotoxic activity of the pulsed splenocytes was measured by the LDH assay.

Statistical analysis. The statistical analysis was performed using one-way analysis of variance. $\mathrm{P}<0.05$ was used to indicate a statistically significant difference.

\section{Results}

Characterization of CD4OL-EXO. The morphology of the exosomes that were obtained was examined first. Under electron microscopy, the exosomes revealed the typical characteristics of a round morphology, with a diameter of 30-90 nm (Fig. 1A). Western blot analysis demonstrated that all the types of exosomes contained the exosome-associated proteins, HSP70, TSG101, CD63 and CD9 (Fig. 1B). In total, 10 $\mu \mathrm{g}$ exosomal proteins was routinely obtained from supernatants containing $1 \times 10^{7} 3 \mathrm{LL}$ cells. There was no marked difference in the production of exosomes from the Ad-CD40L and Ad-Lac Z-infected and uninfected 3LL cells. The CD40L was then detected on each type of exosome by FACS. CD40L was only detected on CD40L-EXO, but not Lac Z-EXO or Control-EXO (Fig. 1C). These results indicate that the exosomes carrying CD40L are likely to possess a potent ability to induce the activation of DCs were successfully isolated. 
A
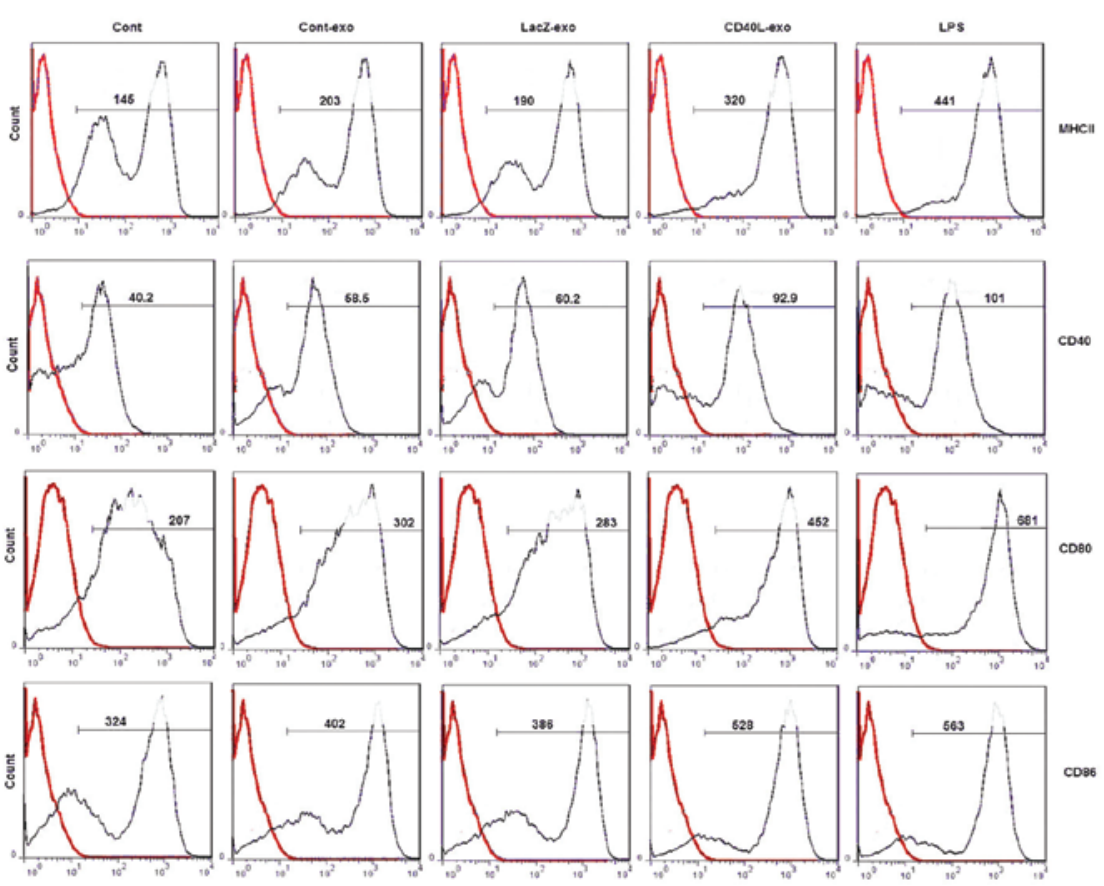

B
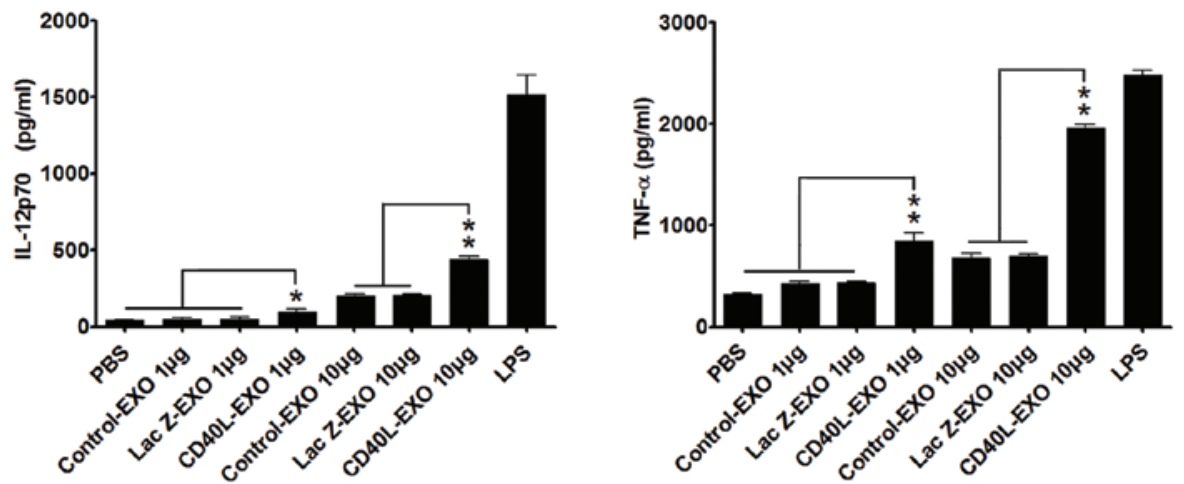

C

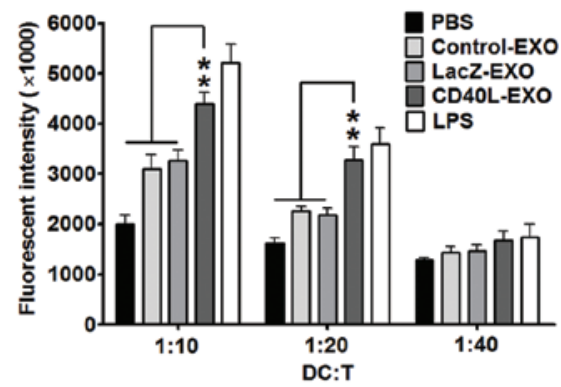

D

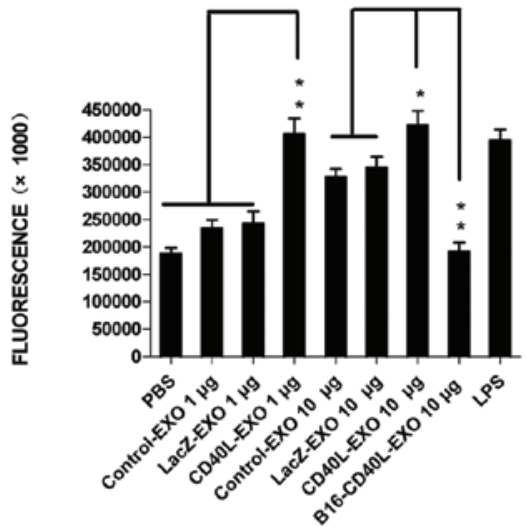

Figure 2. CD40L-EXO induce activation of BMDCs more efficiently. (A) FACS analysis of the BMDC phenotype. BMDCs were stimulated with each type of exosome (1 or $10 \mu \mathrm{g} / \mathrm{ml})$ or LPS $(1 \mu \mathrm{g} / \mathrm{ml})$. Following $48 \mathrm{~h}$ of incubation, the cells were collected and stained with isotype (broken line) or PE-labeled anti-MHC-II, CD40, CD80 and CD86 (solid line) monoclonal antibody, washed and analyzed by FACS. The numbers indicate the mean fluorescence intensity. The data are representative of three independent experiments. (B) In parallel, cytokines in the supernatants were detected by ELISA. (C) CD40L-EXO increased the ability of DCs to stimulate allogeneic splenic CD4 ${ }^{+} \mathrm{T}$ cell proliferation in MLR. Following co-culture with each type of exosome for $48 \mathrm{~h}$, BMDCs were collected and inactivated by mitomycin $\mathrm{C}$. The splenic CD4+ ${ }^{+}$cells from BALB/c mice were cultured with the pre-treated C57BL/6 DCs at the indicated ratios for $96 \mathrm{~h}$. The proliferation of $\mathrm{T}$ cells was determined by the alamarBlue assay ( $\mathrm{n}=5$ ). (D) Tumor antigen-specific CD $4^{+} \mathrm{T}$ cell proliferation induced by CD40L-EXO. C57BL/c mice were immunized with 3LL cell lysates. CD4 ${ }^{+} \mathrm{T}$ cells were purified from the spleen of immunized mice on day seven and co-cultured with syngeneic BMDCs that were pretreated with each type of exosome (1 or $10 \mu \mathrm{g} / \mathrm{ml})$. Following a total $96 \mathrm{~h}$ of culture, the proliferation of $\mathrm{CD}^{+} \mathrm{T}$ cells was also determined by the alamarBlue assay $(\mathrm{n}=5)$. Data are representative of three independent experiments. $\mathrm{P}<0.05$ and ${ }^{* *} \mathrm{P}<0.01$. CD40L-EXO, CD40 ligand gene-modified 3LL tumor cells; BMDC, bone marrow-derived dendritic cells; FACS, fluorescence activated cell sorting; MLR, mixed lymphocyte reaction; DC, dendritic cells; LPS, lipopolysaccharide; PE, phycoerythrin.. 

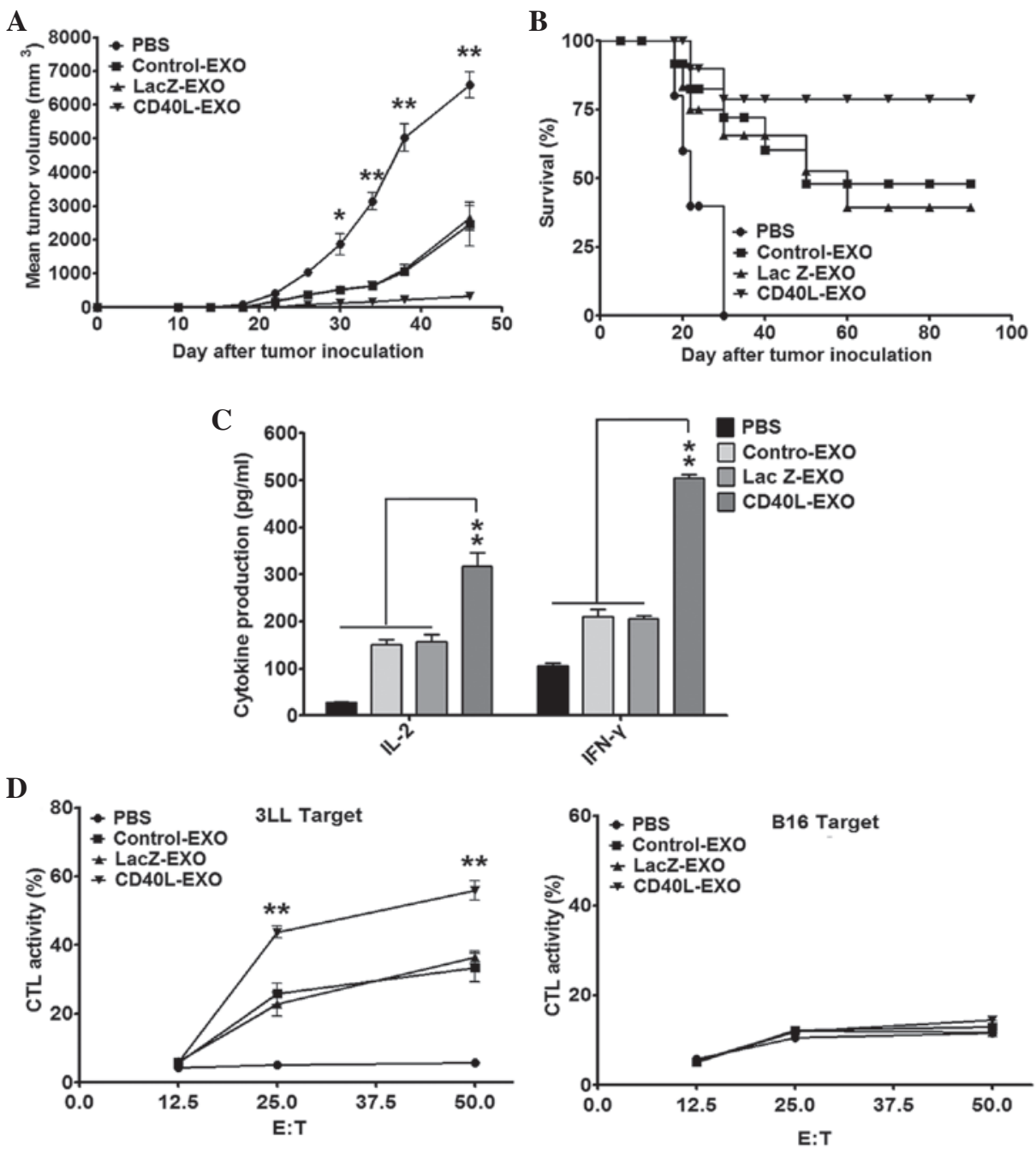

Figure 3. CD40L-EXO induce a stronger protective antitumor immunity. Tumor development in CD40L-EXO-immunized mice. (A) Tumors were established in C57BL/6 mice ( $\mathrm{n}=10 / \mathrm{group}$ ) by immunization four times by s.c. injection with PBS and $10 \mu \mathrm{g}$ of each type of exosome on days $-13,-11,-9$ and -7 . On day 0 , the mice received a s.c. injection of $1 \times 10^{6} 3 \mathrm{LL}$ cells. The tumor size was monitored with a caliper on the indicated days. (B) The tumor-free mice were also recorded. (C) The levels of IFN- $\gamma$ and IL-2 secreted from splenocytes of C57BL/6 mice immunized with CD40L-EXO. Mice (n=10) of different groups were sacrificed 7 days following the last immunization. Splenocytes $\left(5 \times 10^{6} / \mathrm{ml}\right)$ were stimulated with inactivated 3LL cells at a ratio of 10:1 for $48 \mathrm{~h}$. The levels of IFN- $\gamma$ and IL-2 in the culture supernatants were measured by ELISA. (D) CTL activity induced by CD40L-EXO immunization. Splenocytes from immunized mice were stimulated with inactivated 3LL cells and $\mathrm{mIL}-2(30 \mathrm{U} / \mathrm{ml})$ for 7 days in vitro, and then the cytotoxicity of pulsed splenocytes to $3 \mathrm{LL}$ target cells or syngeneic B16 cells that served as a specificity control were determined by the LDH assay $(n=5)$. Data are representative of three independent experiments. ${ }^{*} \mathrm{P}<0.05$ and ${ }^{* *} \mathrm{P}<0.01$ vs. PBS, Control-EXO or Lac Z-EXO. CD40L-EXO, CD40L gene-modified 3LL tumor cells; CTL, cytotoxic T lymphocytes; s.c., subcutaneously; E:T, effect cells : target cells; mIL-2, mouse interleukin 2.

CD4OL-EXO induce activation of BMDCs more efficiently. Exosomes from heat-stressed and IL-18- or IL-2 gene-modified tumor cells were more efficient in promoting DC maturation $(8,16,17)$. Considering the significance of CD40 signaling in the immune function of DCs, the phenotype of DCs treated with CD40L-EXO was examined first. Following stimulation with $10 \mu \mathrm{g}$ of all the types of exosomes for $48 \mathrm{~h}$, the expression of MHC II, CD80, CD86 and CD40 on the BMDCs was markedly increased. However, the effect of CD40L-EXO was strongest, which was comparable with the effect of LPS (Fig. 2A). IL-12p70 and TNF- $\alpha$ production in the culture supernatants of the BMDCs was markedly enhanced by CD40L-EXO and was significantly higher than that of Lac Z-EXO and Control-EXO (Fig. 2B). In the MLR, the CD40L-EXO-, Lac Z-EXO- and Control-EXO-treated DCs all induced T cell proliferation. However, DCs stimulated with CD40-EXO were more effective in stimulating a proliferative response than those with Lac Z-EXO and Control-EXO (Fig. 2C). Furthermore, it was also determined whether DCs pulsed with CD40L-EXO, Lac Z-EXO and Control-EXO had differential abilities to induce the activation of tumor antigen-specific $\mathrm{CD} 4^{+} \mathrm{T}$ cells from mice immunized with $3 \mathrm{LL}$ cell lysates. The proliferation of the primed $\mathrm{CD} 4^{+} \mathrm{T}$ cells induced by the DCs treated with CD40L-EXO was greater than those treated with Lac Z-EXO and Control-EXO. Notably, TEX from the CD40 gene-modified B16 tumor cells hardly induced the proliferation of the primed $\mathrm{CD} 4^{+} \mathrm{T}$ cells (Fig. 2D). These data reveal that CD40L-EXO are more effective in inducing the phenotypic and functional maturation of DCs than Lac Z-EXO and Control-EXO, and CD40L-EXO may also induce a more efficient tumor antigen-specific $\mathrm{CD} 4^{+} \mathrm{T}$ cell immunity.

CD4OL-EXO induce a stronger protective antitumor immunity. To determine the antitumor immunity induced by 
A

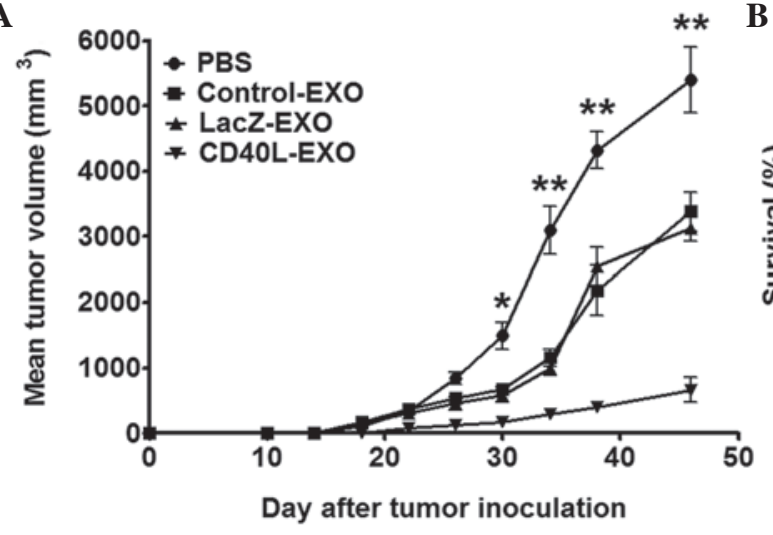

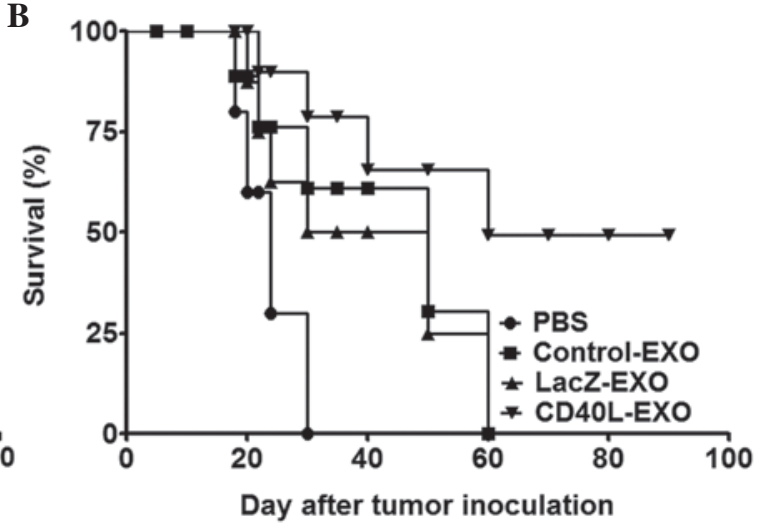

Figure 4. CD40L-EXO induce a stronger therapeutic antitumor immunity. C57BL/6 mice (n=10/group) were pre-inoculated with $1 \times 10^{6} 3 \mathrm{LL}$ tumor cells Following 7 days of tumor inoculation, the mice received s.c. injections of $10 \mu \mathrm{g}$ of each type of exosome at days $+7,+9,+11$ and +13 . (A) The tumor size was monitored with a caliper at the indicated days. (B) The tumor-free mice were also recorded. The data are presented as the mean \pm SD from three independent experiments; ${ }^{*} \mathrm{P}<0.05$ and ${ }^{* *} \mathrm{P}<0.01$ vs. phsophate-buffered saline (PBS), Control-EXO or Lac Z-EXO. CD40L-EXO, CD40L gene-modified 3LL tumor cells; s.c., subcutaneously.

CD40L-EXO, the mice were immunized with each type of exosome and challenged s.c. with $1 \times 10^{6} 3 \mathrm{LL}$ cells. The immunization of CD40L-EXO resulted in a significantly enhanced antitumor effect when compared with the Lac Z-EXO-, Control-EXO- or PBS-treated mice (Fig. 3A). The survival rate of the tumor mice immunized with CD40L-EXO was $\sim 80 \%$ on day 90 , but only 40 and $50 \%$ in the tumor mice immunized with Lac Z-EXO and Control-EXO, respectively (Fig. 3B). As predicted, treatment with PBS revealed no significant protection against the inoculated 3LL cells (Fig. 3A and B). These results indicate that $\mathrm{CD} 40 \mathrm{~L}-\mathrm{EXO}$ induce a stronger protective antitumor immunity than unmanipulated exosomes.

CTLs are important in the eradication of tumor cells. Effective cytokines, including IFN- $\gamma$ and IL-2, are extremely important in the antitumor immunity of CTLs. The levels of IFN- $\gamma$ and IL- 2 were detected from the splenocytes of the exosomes from the immunized mice following restimulation with 3LL cells ex vivo. Immunization with all exosomes significantly increased the production of IFN- $\gamma$ and IL-2 from the splenocytes. However, the effect of CD40L-EXO was the strongest (Fig. 3C). Furthermore, the cytotoxic activity of the splenocytes from the CD40L-immunized mice to 3LL targets was more potent than that of the splenocytes from Lac Z-EXO-, Control-EXO- or PBS-immunized mice (Fig. 3D). These results indicate that immunization with CD40L-EXO induces more robust Th1 and antigen-specific CTL responses.

CD4OL-EXO induce a more powerful therapeutic antitumor immunity. To further confirm the antitumor potential of CD40L-EXO, the present study examined whether CD40L-EXO were effective in eradicating the established tumor in mice. Mice were inoculated with 3LL cells on day 0. Following the formation of visible tumors, as observed by the naked eye, the mice were treated with each type of exosomes (10 $\mu \mathrm{g} /$ mouse) by s.c. injection on days $+7,+9,+11$ and +13 . As predicted, tumor growth in the mice treated with CD40L-EXO was effectively inhibited, with $50 \%$ of the mice surviving up to day 90 (Fig. 4). These results demonstrate that CD40L-EXO have a superior antitumor effect compared with Lac Z-EXO and Control-EXO.

\section{Discussion}

With recent scientific and technological progress, the development of tumor immunotherapy has significantly improved the prognosis of cancer patients, but the reality of the "war on cancer' remains ongoing (18). A number of technical challenges restrict the effect of tumor immunotherapy in the clinic. For example, tumor antigens often exhibit poor immunogenicity. Tumor cells frequently escape the immune response via variation. Exosomes, as potential therapeutic agents for tumors, have attracted the intensive attention of oncologists $(19,20)$. However, the efficacy of traditionally prepared exosomes in the induction of antitumor responses remains to be optimized. In the present study, exosomes from CD40L gene-modified 3LL lung tumor cells were isolated, and it was revealed that CD40L-EXO were more efficient in the induction of antitumor $\mathrm{T}$ cell immunity.

Exosomes have been tested in three Phase I clinical studies in melanoma, lung and colorectal cancer patients (19-21). These studies demonstrated that the safety of administering exosomes in humans was satisfactory. Clinical observations indicated that exosomes may stimulate the adaptive and innate cellular immune responses. Regardless of the observation of positive antitumor responses, the effect of exosomes in tumor therapy is not yet ideal. It has been reported that TEX from immune-activated gene-modified tumor cells and heat-stressed tumor cells or super-antigen anchored TEX demonstrated potent antitumor effects $(8,9,16)$. Similarly, it was hypothesized that TEX from CD40L gene-modified tumor cells also have enhanced the immunostimulatory potential. This hypothesis was suggested to be valid by the findings of the present study, which showed that CD40L-EXO more efficiently induced DC activation and the subsequent priming of antitumor $\mathrm{T}$ cell immunity.

DCs are important in the initiation of antitumor $\mathrm{T}$ cell immunity, which requires the full activation of DCs. CD40 molecules are expressed on the immature DCs and are impor- 
tant in their activation. Following maturation mediated by CD40 signaling, DCs may effectively prime antigen-specific $\mathrm{CD}^{+}$and $\mathrm{CD}^{+} \mathrm{T}$ cell responses (14). However, CD40L is predominantly expressed on activated $\mathrm{T}$ cells, but not on resting $\mathrm{T}$ cells, which is contradictory and inefficient for the activation of DCs. CD40L-EXO-containing tumor antigens were rich in CD40L, which may have efficiently activated the DCs, resulting in the activation of tumor antigen-specific $\mathrm{T}$ cells. The activated $\mathrm{T}$ cells upregulated the expression of CD40L and further activated the DCs, which possibly led to a positive-feedback for tumor antigen-specific $\mathrm{T}$ cell activation.

It has been reported that TEX from heat-stressed 3LL lung tumor cells induce strong antitumor effects. These exosomes have been demonstrated to effectively chemoattract DCs to the tumor site (23). The accumulation of DCs in the lungs is beneficial to prime a robust antitumor $\mathrm{T}$ cell immunity. It also has been reported that DCs infiltrating human non-small cell lung cancer are blocked at an immature stage (22). In lung cancer, the function of DCs is converted from immune activation to tolerance. CD40L-EXO have the potent ability to activate DCs, which possibly turns over the tolerance of DCs. It may be supposed that TEX from heat-stressed and CD40L-gene modified 3LL lung tumor cells is likely to increase the numbers of DCs in the tumor site and enhance their activation. Therefore, TEX prepared from heat-stressed and CD40L-gene modified lung tumor cells may be more promising for the treatment of lung cancers.

Altogether, in the present study, CD40L-EXO were isolated from 3LL tumor cells infected by Ad-CD40L and it was demonstrated that CD40L-EXO were more efficient in $\mathrm{DC}$ activation and antitumor $\mathrm{T}$ cell immunity induction. As cell-free vesicles, exosomes are stable for a long period of time when preserved at $-80^{\circ} \mathrm{C}$. The production of TEX is extremely large-scale and the cost for TEX preparation is economical. Moreover, due to TEX often carrying a low number of or no MHC molecules, the biological safety of TEX is ideal. All these characteristics render CD40L-EXO a prospective universal agent for the therapy of lung cancer.

\section{Acknowledgements}

This study was supported by the National Natural Science Foundation of China (grant no. 81200014), the Natural Science Foundation of Zhejiang Province (grant no. LY12H13003) and the Medicine and Health Foundation of the Health Bureau of Zhejiang Province (grant nos. 2012KYA152 and 2010KYA104).

\section{References}

1. Sugimura H, Nichols FC, Yang P, et al: Survival after recurrent nonsmall-cell lung cancer after complete pulmonary resection. Ann Thorac Surg 83: 409-418, 2007.

2. Théry C, Zitvogel L and Amigorena S: Exosomes: composition, biogenesis and function. Nat Rev Immunol 2: 569-579, 2002.
3. Lamparski HG, Metha-Damani A, Yao JY, et al: Production and characterization of clinical grade exosomes derived from dendritic cells. J Immunol Methods 270: 211-226, 2002.

4. Chaput $\mathrm{N}$ and Théry $\mathrm{C}$ : Exosomes: immune properties and potential clinical implementations. Semin Immunopathol 33: 419-440, 2011.

5. Ohshima K, Inoue K, Fujiwara A, et al: Let-7 microRNA family is selectively secreted into the extracellular environment via exosomes in a metastatic gastric cancer cell line. PLoS One 5: e13247, 2010.

6. Zhang J, Zhang Y, Luo C, Xia Y, Chen $\mathrm{H}$ and $\mathrm{Wu} \mathrm{X}$ : Glycosyl-phosphatidylinositol-anchored interleukin-2 expressed on tumor-derived exosomes induces antitumor immune response in vitro. Tumori 96: 452-459, 2010

7. Zhang Y, Wu XH, Luo CL, Zhang JM, He BC and Chen G: Interleukin-12-anchored exosomes increase cytotoxicity of T lymphocytes by reversing the JAK/STAT pathway impaired by tumor-derived exosomes. Int J Mol Med 25: 695-700, 2010.

8. Dai S, Wan T, Wang B, et al: More efficient induction of HLA-A*0201-restricted and carcinoembryonic antigen (CEA)-specific CTL response by immunization with exosomes prepared from heat-stressed CEA-positive tumor cells. Clin Cancer Res 11: 7554-7563, 2005.

9. Xiu F, Cai Z, Yang Y, Wang X, Wang J and Cao X: Surface anchorage of superantigen SEA promotes induction of specific antitumor immune response by tumor-derived exosomes. J Mol Med (Berl) 85: 511-521, 2007.

10. Armitage RJ, Fanslow WC, Strockbine L, et al: Molecular and biological characterization of a murine ligand for CD40. Nature 357: 80-82, 1992.

11. Melief CJ: Cancer immunotherapy by dendritic cells. Immunity 29: 372-383, 2008

12. Dohnal AM, Luger R, Paul P, Fuchs D and Felzmann T: CD40 ligation restores type 1 polarizing capacity in TLR4-activated dendritic cells that have ceased interleukin-12 expression. J Cell Mol Med 13: 1741-1750, 2009.

13. Elmetwali T, Searle PF, McNeish I, Young LS and Palmer DH: CD40 ligand induced cytotoxicity in carcinoma cells is enhanced by inhibition of metalloproteinase cleavage and delivery via a conditionally-replicating adenovirus. Mol Cancer 9: 52, 2010

14. van Kooten C and Banchereau J: CD40-CD40 ligand. J Leukoc Biol 67: 2-17, 2000.

15. Van Nuffel AM, Corthals J, Neyns B, Heirman C, Thielemans K and Bonehill A: Immunotherapy of cancer with dendritic cells loaded with tumor antigens and activated through mRNA electroporation. Methods Mol Biol 629: 405-452, 2010.

16. Dai S, Zhou X, Wang B, et al: Enhanced induction of dendritic cell maturation and HLA-A*0201-restricted CEA-specific CD8(+) CTL response by exosomes derived from IL-18 gene-modified CEA-positive tumor cells. J Mol Med (Berl) 84: 1067-1076, 2006.

17. Chen W, Wang J, Shao C, et al: Efficient induction of antitumor $\mathrm{T}$ cell immunity by exosomes derived from heat-shocked lymphoma cells. Eur J Immunol 36: 1598-1607, 2006.

18. Miller MJ, Foy KC and Kaumaya PT: Cancer immunotherapy: present status, future perspective, and a new paradigm of peptide immunotherapeutics. Discov Med 15: 166-176, 2013.

19. Escudier B, Dorval T, Chaput N, et al: Vaccination of metastatic melanoma patients with autologous dendritic cell (DC) derived-exosomes: results of thefirst phase I clinical trial. J Transl Med 3: 10, 2005.

20. Morse MA, Garst J, Osada T, et al: A phase I study of dexosome immunotherapy in patients with advanced non-small cell lung cancer. J Transl Med 3: 9, 2005.

21. Wei H, Wang H, Lu B, et al: Cancer immunotherapy using in vitro genetically modified targeted dendritic cells. Cancer Res 68: 3854-3862, 2008.

22. Perrot I, Blanchard D, Freymond N, et al: Dendritic cells infiltrating human non-small cell lung cancer are blocked at immature stage. J Immunol 178: 2763-2769, 2007.

23. Chen T, Guo J, Yang M, Zhu X and Cao X: Chemokine-containing exosomes are released from heat-stressed tumor cells via lipid raft-dependent pathway and act as efficient tumor vaccine, J Immunol 186: 2219-2228, 2011. 